\title{
Risks Assessment Before Pregnancy: A Healthy Mother and Baby After Delivery
}

\author{
Article by A. Kadango \\ PhD, Lecturer, University of Malawi, Kamuzu College of Nursing Campus \\ E-mail: alicekadango@kcn.unima.mw
}

\begin{abstract}
Risk assessment before pregnancy is one of the interventions during Preconception care (PCC) that would improve the outcome of pregnancy. A quantitative descriptive non-experimental approach was used to examine the use of preconception interventions in health centres in Blantyre urban. The study assessed the PCC men and women of child bearing age have to ensure a healthy mother and baby after pregnancy. The objectives were to: explore and describe the knowledge men and women of childbearing age have on HTSP and PCC, identify variables that influence men and women to acquire appropriate knowledge on PCC. The study included 300 men and women of child bearing age that attended outpatient clinics for various minor ailments like underfive, family planning and gyneacological clinic who were between 18 to 35 years. Structured questionnaire was used to gather data and descriptive statistics were used to analyse data using SPSS version 20. Kruskal Wallis test and Chi square was used to determine the relationship of specific variables to use of PCC. Most of the respondents were not assessed for chronic problems that would interfere pregnancy like diabetes, hypertension, aneamia and malnutrition. Only $30 \%$ were checked for VDRL, $43 \%$ were vaccinated from tetanus but $74 \%$ were assessed for HIV test. Chronic diseases would be controlled before pregnancy to minimize adverse effects of pregnancy like preterm labour, preeclampsia, intra uterine growth retardation (IUGR) and neonatal infections. Universal education should be provided to men and women of child bearing age to improve the understanding to have risk assessments done before pregnancy.
\end{abstract}

Keywords: Risk assessment; medical assessment; Maternal health; Newborn health; Unintended BMI; Preconception care;

\section{Introduction}

Maternal and neonatal health care remains a challenge globally and there is a need to have strategies that will address the issues affecting women and children. Despite the efforts to improve maternal and newborn care globally, 289,000 women died in 2013 owing to pregnancy (WHO 2015). About 800 maternal deaths occurred in sub-Saharan Africa. WHO (2015) indicates that the number of women dying owing to complications has decreased by $45 \%$ from 523,000 in 1990 to 289,000 in 2013. Steel et al (2015) indicated that there is growing evidence that PCC can improve maternal health prio to conception and directly improve the health of the mother and the fetal environment during pregnancy.

Chronic diseases like diabetes mellitus, hypertension, anaemia, cardiac diseases, respiratory diseases, epilepsy, cancer, and many others also affect men and women of the reproductive age indicating that their pregnancy outcome is affected. Chronic diseases assessment before the onset of pregnancy should be managed to prevent adverse outcomes of pregnancy owing to its effects (Dean et al 2013). Dunlop et al (2013) reported that women with chronic medical conditions have more complications to the mother and the fetus. It is important for these at-risk groups to have proper counselling and management of their conditions before conception to ensure a healthy outcome of pregnancy. Bombard, Robbins, Dietz and Valderrama (2013) emphasized the importance of encouraging women to practice health life styles before conception and screening of diseases like hypertension to reduce adverse effects during pregnancy. Prior to having a pregnancy, couples should be in optimal health to support life of the unborn baby. Uncontrolled chronic diseases have detrimental effects to the outcome of pregnancy to both the mother and the baby (Lassi et al 2014). Vinturache et al (2014) highlighted that obesity is associated with increased complications during pregnancy, labour, delivery and postpartum period. Recommendations that were provided in a summit on PCC resolved that all women of the childbearing 
age have to receive PCC services, screening for chronic diseases so that pregnancy begin when one is in optimal health (Posner et al 2006).

A qualitative research conducted by Tuomeinen et al (2013) stated that women of the childbearing age were able to know that alcohol, tobacco and illicit drugs are dangerous but did not comprehend the importance of accessing health care services to have cervical smears, risk assessment for diseases, treatment review before pregnancy. There is a need for awareness to have a thorough check up to rule out any chronic diseases and their treatment that could have adverse effect towards pregnancy. PCC could be provided at primary care level so that couples are screened of the health problems that would affect them (Tuomeinen et al 2013). At primary level of health provision, PCC offers an opportunity to address risk factors that would affect prospective parents throughout the life span (Salihu et al 2013). Mason et al (2014) conclude that if PCC is practised medical costs will be minimised as the complications that would have developed are controlled before occurrence. Most people like to consult the health care providers when complications occur which is costly. Health assessment conducted on prospective parents is beneficial to prevent complications that would occur. Even in the western world there is at least $20 \%$ of adverse effects of pregnancy like miscarriage, still birth, preterm birth, low birth weight or congenital abnormality (Elsinga et al 2006). PCC like risk assessments before conception would improve the outcome of pregnancy.

In a study done in Malawi on men and women of child bearing age findings indicated that PCC is not provided in most of the facilities. There are no wellness clinics that would offer this service and guidelines are not available for health care providers to follow to provide comprehensive PCC. The guidelines include screening of chronic diseases in men and women of child bearing age including genetic counselling. Maintenance of nutritional status of a child bearing mother is essential to yield a healthy outcome of the mother and the baby. Prophylactic treatment like folic acid should be provided before pregnancy to prevent neural tube defects. Vaccination from tetanus should be done before conception to protect the baby to be born.

\section{Statement of the research problem}

The consequences of late contact to health care lead to adverse effect that are detrimental to maternal and newborn outcome. The high maternal and neonatal deaths are persisting despite interventions. Provision of micronutrients, nutrition counselling, screening of chronic diseases, management of medical conditions, and prevention of risky behaviours such as cigarette smoking and alcohol intake during PCC can help to reduce maternal and neonatal morbidity and mortality. Knowledge on the importance of PCC motivates clients to seek PCC services. In Malawi, there are no standardised guidelines that include interventions to improve the provision of PCC. If women have knowledge on PCC it makes them to be proactive to indulge in health care activities that would improve the outcome of pregnancy. Some of the interventions to improve maternal and newborn health include early assessments of medical conditions that would affect pregnancy, early treatment of chronic condition that can stabilize before conception, prophylactic treatment and vaccinations from some infections that can affect the newborn should be controlled.

\section{Purpose of the study}

The research purpose was to identify the PCC that is available in Malawi and identify the gaps that exist and develop appropriate guidelines and strategies that would assist to improve maternal mortality rate that is very high in Malawi. Preconception care is not highly emphasised in provision of maternal and newborn health that can assist to reduce the detrimental effects during pregnancy to have a healthy mother and baby.

\section{Objectives of the study}

- Explore and describe the level of knowledge men and women of childbearing age have on HTSP/PCC.

- Develop relevant preconception interventions that would assist to improve maternal and newborn outcomes in developing country like Malawi. 


\section{Risk assessments before conception}

Most men and women of childbearing age are challenged with various conditions affecting pregnancy like medical diseases, surgical conditions gynaecological problems, and infections and the problem related with nutrition. These conditions need to be assessed before attempting to get pregnancy. The study conducted in Blantyre urban indicated that $30 \%$ of respondents had anaemia, hypertension and respiratory problems. PCC requires collaboration of health care providers so that it can assess problems, manage and refer to specialists if necessary (Poels et al 2017). The role of different cadres of health care providers in providing PCC is to provide the service or identify a problem and refer. Anderson et al (2006) highlight that as clients come to contact with the health care provider, it is an opportunity to administer PCC.A study done in Ethiopia indicated that only $27 \%$ of the respondents had knowledge on preconception care (Ayalew et al 2017).

\section{Screening for infectious and chronic diseases that affect maternal and infant birth outcomes}

Couples intending to get pregnant need appropriate screening and treatment of diseases that would affect pregnancy. Mahmud and Mazza (2010) indicated that conditions like diabetes in pregnancy is associated with higher rate of miscarriage, preeclampsia, preterm labour and fetal malformation. The clinical components of PCC include taking family history, behaviours, obstetric history, screening for diseases, genetic disorders and counselling on folic acid consumption, smoking, alcohol intake and nutrition history (Salihu et al 2013). It is possible to conduct a preconception risk assessment among couples of reproductive age. A research conducted by Gardiner et al (2013) indicate that almost all women thought that preconception risk assessment was useful to identify the risks before pregnancy like screening for sexually transmitted diseases, adequate nutrition during pregnancy and appropriate multivitamin supplementation with folic acid. Further qualitative study conducted in the UK revealed that younger women were less aware on preconception health and the women could know the prevention of smoking, alcohol and illicit drugs but could not see the importance of checking immunisations, medications, family history, ruling out diseases, and taking vitamin supplements prior to conceptions (Tuomainen et al 2013). Folic acid supplementation was taken by any woman of reproductive age that decides to get pregnant in order to have the prevention of neural tube defects on the newborn babies in the US. This is one of the interventions that were done prior to conception in the 90s (Waggoner 2012). The Centre for Disease Control (CDC) had a summit with 35 countries to develop goals, recommendations and action steps to strengthen provision of preconception health to improve pregnancy outcomes (Posner et al 2006). One of the recommendations was to strengthen the prepregnancy check up to couples and individuals planning to get pregnant so that are screened and treated on any diseases that have adverse effect to the pregnancy outcome (Posner et al 2006). Controlling known medical problems prior to pregnancy does not only help to optimise the mother's health but it may also improve neonatal and paediatric outcomes (Curtis et al 2006).

\section{Screening for syphilis, chlamydia and gonococcal infections}

PCC provides an opportunity when there is time to screen and treat infectious diseases that can affect the foetus in the utero. Lassi et al (2014) underscore that increasing awareness to have screening and treatment of STIs and HIV will significantly promote newborn health. Prenatal care is too late to improve birth outcome because many of the interventions to prevent adverse maternal and infant outcomes are not administered soon enough to have an effect. Preconception and prenatal care together can improve perinatal outcome, as mothers will conceive at the right time when the mother is in good health. Syphilis is caused by a bacterium called traponema pallidum that if the mother is not treated in pregnant, there is $100 \%$ chance to infect the unborn baby. There is also $50 \%$ chance that the infected baby would die shortly before or after birth. The baby that survives would have low birth weight, failure to thrive and even long-term neurological problems like blindness and deafness. The bacterium that has affected the pregnant mother has ability to cross the placenta and affect the unborn baby. Sagel et al (2011) explained that fetal infections and complications can be prevented by initiating screening programs which could be done before onset of pregnancy. It is important that couples and individuals intending to become prospective parents need to screened from transplacental infections that would affect foetal development. Chlamydia infections should be screened and treated as they could affect 
baby's eyes, ears and genitals. Gonorrhoea can be passed to the baby during childbirth and could affect the eyes of the baby causing gonococcal neonatorum that can lead to blindness. More importantly, PCC would prevent occurrence and treat these infections that affect newborn in the utero as well as at birth. Programmes should be put in place in countries like Malawi to provide PCC that would reduce neonatal infections that cause morbidity and mortality of infants.

\section{Research methodology}

\section{Study design, site and population}

The study used a quantitative descriptive design to determine the preconception care that is provided in a Malawian setting. A questionnaire adapted from a study done in Texas on PCC was used to assess the utilization of preconception care in the health centres in urban Blantyre. The total sample was 302 men and women of childbearing age that had a pregnancy before and intend to get pregnant in the next five years.

Quantitative research method was used to measure the concept of PCC that is provided in Malawian health care settings and identify the gaps that exist to improve the care before pregnancy in order to yield healthy outcomes of the mother and the baby (Bryman 2012). Data was collected using a developed questionnaire adapted from a study done in Texas on preconception care and results were analysed using statistics. The research used quantitative approach to investigate what is available on the ground on the care that is provided before conception to have a healthy outcome of the mother and the baby. In order to generate information a survey had to be done on the selected sample that provided information and later the findings were generalised to the study population. The relevant strategies will assist policy makers to improve on provision of care that would assist individuals to have improved outcomes of pregnancy.

\section{Sample size}

A sample size was calculated using the number of clients that attended the three clinics. The clinics used were the ones accessible to the researcher and had enough clientele that get health care services. Kothari (2004) states that technically the sample size should be large enough to give a confidence interval of desired width that can be representative to the population. Other factors considered when drawing the sample was the nature of the units, size of the population, size of the questionnaire, availability of trained investigators and the time available to conduct the study.

A sample size was a representative of the total population and helped to generalize research findings to the entire population. Solvin formula was be used to help to calculate a sample from a known population (Andale 2012 at www.statisticshowto.com/-how to use- Slovins formula accessed $1^{\text {st }}$ April 2016.

\section{Sampling}

Probability sampling method was used to allow each member to have the chance to be chosen for the sample so that there is representativeness to the population to develop appropriate interventions in PCC that would be applicable to the people before conception. Quantitative research use stratified random sampling to have subjects that are homogenous in relation to study variables that represent the total sample. The researcher used probability sampling method to identify participants booked for their visit at the health care clinic.

The inclusion criteria included men and women of the reproductive age that are intending to get pregnant between the ages of 18 to 35 years of age with at least one child. The sample from an accessible population was selected to represent the population so that the results can be generalised to the target population. A sampling plan that specifies in advance how participants are selected was done in advance before sampling (Polit $\&$ Beck 2012). The number of respondents of child bearing age was 300 from the three clinical facilities sampled.

\section{Instruments}

The instrument used in this study were adapted from a study done in Texas and comprised of five sections: demographic data, psychological data, reproductive health data risk assessments before 
conception and screening from health hazards. Pre-test of the questionnaire was done at Queen Elizabeth Central Hospital and results were not included in the study results. Data collectors were recruited from nurses and midwives depending on their availability for training and data collection. Data collectors needed to be fluent in English and Chichewa language. Data collectors were trained on the purpose of the research, how to collect data and ethics observed when conducting the study. The face to face interviews using a structured questionnaire lasted 30 minutes.

The instrument was reviewed by reproductive health specialists and midwives who ensured that the questions addressed pertinent issues that would address preconception care. A statistician was consulted who refined the questionnaire so that items can be easily be computed using SPSS version 20.0. The College of medicine Research Ethics Committee requested the validation of the questionnaire and an instrument used in a study done in Texas was used and adapted to include other aspects to be examined on preconception care.

\section{Reliability and validity}

The questionnaire was adapted from a study done in Texas and previous testing showed that it was reliable, with good predictive validity and sensitivity. The Cronbach alpha coefficient was 0.657 for all the items and a Spearman rank correlation coefficient of $0.817(\mathrm{p}<0.001)$. The scale was translated by a Chichewa language specialist and modified by a reproductive specialist who ensured that the content examined what it is intending to measure.

\section{Analysis}

The numerical data were analysed using computerised statistical Package for Social Sciences (SPSS) version 20.0. Findings were displayed in tables, charts, frequency distribution tables, proportions and percentages. Cross tabulations were done inoder to visualise summarized data related to two variables within the study sample. The significance of association between variables was done using chi square $\left(\mathrm{X}^{2}\right)$ test as $\mathrm{P}$ values were calculated to evaluate whether a relationship between the variables are statistically significant. The level of significance was set at .05 or .01. Fisher exact and Kruskal Wallis test were used to assess where there is a significant association between a specific variable with the clients' knowledge on preconception care. Analysis of variance was used to investigate association between continuous variable and knowledge on preconception care.

\section{Ethical consideration}

The higher degrees committee of the Department of Health Studies at the University of South Africa and College of Medicine Ethical Committee reviewed the proposal and granted permission to conduct the study. An instrument from a study done in Texas on preconception care was adapted to enrich the questionnaire with specific content to measure in preconception care.

Permission was granted to conduct the study on study sites from the Deputy Hospital Director of Queen Elizabeth Hospital where the pilot study was done and the District Health Officer for Blantyre urban health centers where the study was done.

Recruitment of research assistants was done and these were Nurses and Midwives that had time to attend to the training and collect data. Training was done on the purpose of the study and its objectives, how to collect data using the questionnaire, proper handling of respondent with respect and dignity and record keeping.

During the interview day respondents were informed of the purpose of the study and its benefits. The respondents were provided with an informed consent to participate in the study. They were informed that they were free to withdraw for participating from the study at any time in course of the interview without any penalty. Those that were interviewed late and came from very far were provided with lunch allowance and transport.

Audio and visual privacy was maintained throughout the interview process. No hazards were encountered during the data collection process. All questionnaires were coded and no name was endorsed on the interview form to maintain confidentiality of the information. The supervisor ensured that all the questionnaires are completed and were kept secure in a lockable suitcase to protect them 
from the rains and missing them. A qualified data entry officer entered all data in the software and it was kept on a secure password which was accessible to the author and the statistician.

\section{Results}

Most men and women of childbearing age are challenged with various conditions affecting pregnancy like medical diseases, surgical conditions gynaecological problems, and infections. These conditions need to be assessed before attempting to get pregnant. During the study that was conducted, men and women who conducted an assessment, 30\% were from assessments from anaemia, hypertension and respiratory problems. PCC requires collaboration of health care providers so that some ailments can be assessed, managed and referred to specialists if necessary (Poels 2017). The role of different cadres of health care providers in providing PCC is to provide the service or identify a problem and refer. Anderson (2006) highlight that as clients come to contact with the health care provider, it is an opportunity to conduct a thorough health assessment and counsel on PCC.A study done in Ethiopia indicated that only $27 \%$ of the respondents had knowledge on preconception care (Ayalew 2017).

Table 1. Risk assessment before conception

\begin{tabular}{|l|l|l|}
\hline \multirow{2}{*}{ Risk assessments done before conception } & Yes & No \\
\cline { 2 - 3 } & $\mathbf{n}(\boldsymbol{\%})$ & $\mathbf{n}(\boldsymbol{\%})$ \\
\hline Conditions affecting pregnancy: & & \\
\hline • Respiratory problems & $96(31.8)$ & $206(68.2)$ \\
\hline - Cardiac disease & $33(10.9)$ & $269(89.1)$ \\
\hline - Hypertension & $96(31.8)$ & $206(68.2)$ \\
\hline - Anaemia & $101(33.4)$ & $201(66.6)$ \\
\hline - Malnutrition & $77(25.5)$ & $225(74.5)$ \\
\hline - Abdominal surgery & $15(5.0)$ & $287(95)$ \\
\hline - Gyneacological problem & $52(17.2)$ & $250(82.8)$ \\
\hline - Reproductive health & $59(19.5)$ & $243(80.5)$ \\
\hline - Diabetes & $30(9.9)$ & $272(90.1)$ \\
\hline - Nutritional assessment & $130(43)$ & $171(56.6)$ \\
\hline
\end{tabular}

The number of respondents that were assessed for respiratory problems were $\mathrm{n}=96(31.8 \%)$ while $\mathrm{n}=206(68.2 \%)$ were not assessed. Only $\mathrm{n}=33(10.9 \%)$ were assessed for cardiac diseases while the rest $\mathrm{n}=269(89.1 \%)$ were not assessed. About $\mathrm{n}=96(31.8 \%)$ were assessed for hypertension while $\mathrm{n}=206$ (68.2\%) were not assessed. In addition, $\mathrm{n}=101(33.4 \%)$ were assessed for anaemia while $\mathrm{n}=201(66.6 \%)$ were not assessed. About $\mathrm{n}=77(25.5 \%)$ were not assessed for malnutrition while $\mathrm{n}=225(74.5 \%)$ were not assessed. Only $n=15(5.0 \%)$ were checked if they have abdominal surgery before conception while $\mathrm{n}=287(95 \%)$ were not assessed. Thirty $(9.9 \%)$ of the respondents were assessed for reproductive health problems before conception while $n=272(90.1 \%)$ were not done. In addition, $n=30$ (9.9\%) were assessed for diabetes. An overwhelming majority $(n=272 ; 90.1 \%)$ were not assessed for diabetes. One hundred and thirty (43\%) were assessed for nutritional problems while $\mathrm{n}=171(56.6 \%)$ were not assessed (see Table 1.1).

\section{Assessments of conditions affecting conception}

A thorough assessment should be conducted to men and women of childbearing age to identify risks that could cause complications after conception. Lassi et al (2014) state that all chronic diseases like diabetes and hypertension should be controlled before conception. In the research study conducted, more than $90 \%$ of the respondents were not assessed on diabetes despite being a rising condition in the developing countries. Moreover, Lu et al (2006) maintain that gestational diabetes has increased the risk of macrosomia, birth trauma, newborn hypoglycaemia, and hyperbilirubinemia. During pregnancy, women can develop a dysglycemic state known as gestational diabetes which has an impact to the off spring (Swaleh 2015). Diabetes control before pregnancy is a significant intervention that reduces the occurrence of congenital malformations by $70 \%$ (Lassi et al 2014). All patients that are diagnosed with diabetes need glycaemic control through self-assessment skills, physician directed assessment, care of 
disease and complications, counselling about diet, exercise, and reproductive advice before conception (Lassi et al 2014). Godfrey (2017) conclude that there are consequent benefits for the infant and the mother if maternal nutrition and glycaemic control is instituted before and during pregnancy. Optimising preconception nutrition is significant to avoid major risks like macrosomia (Godfrey et al 2017).

\section{Investigations done before conception}

Most of the respondents were HIV tested $(n=222 ; 89.7 \%)$ while $n=79(26.2 \%)$ were not HIV tested. Furthermore, $n=94(31.1 \%)$ of the respondents were VDRL tested while $n=207(68.5 \%)$ were not VDRL tested. On malaria parasites, $n=90(31.1 \%)$ of the respondents were tested while $n=211(69.9 \%)$ were not tested). Cervical cancer test was done by $n=65$ (21.5\%) of the respondents while 236 did not do any test. Blood group and full blood count was done by $n=38(12.6 \%)$ of the respondents only while $\mathrm{n}=263$ (87.1) did not do any test on blood group and full blood count (see Table 5.6 and Figure 1.1).

\section{Medications to prevent complications during pregnancy}

Only $n=32(10.6 \%)$ of the respondents took folic acid before conception but $n=268(88.7 \%)$ did not have any folic acid before conception. In addition, $n=103(34.1 \%)$ of the respondents took ferrous sulphate while $\mathrm{n}=197(65.20 \%)$ did not take any medication like ferrous sulphate before pregnancy. Furthermore, $\mathrm{n}=106(34.1 \%)$ had taken Fansidar during pregnancy to prevent malaria while $\mathrm{n}=193$ (63.9\%) did not take Fansidar. Only $\mathrm{n}=49$ (16.2\%) had multivitamins to improve appetite during pregnancy while $n=251$ (83.7\% did not have any of the multivitamins (see Table 5.6 and Figure 5.19).

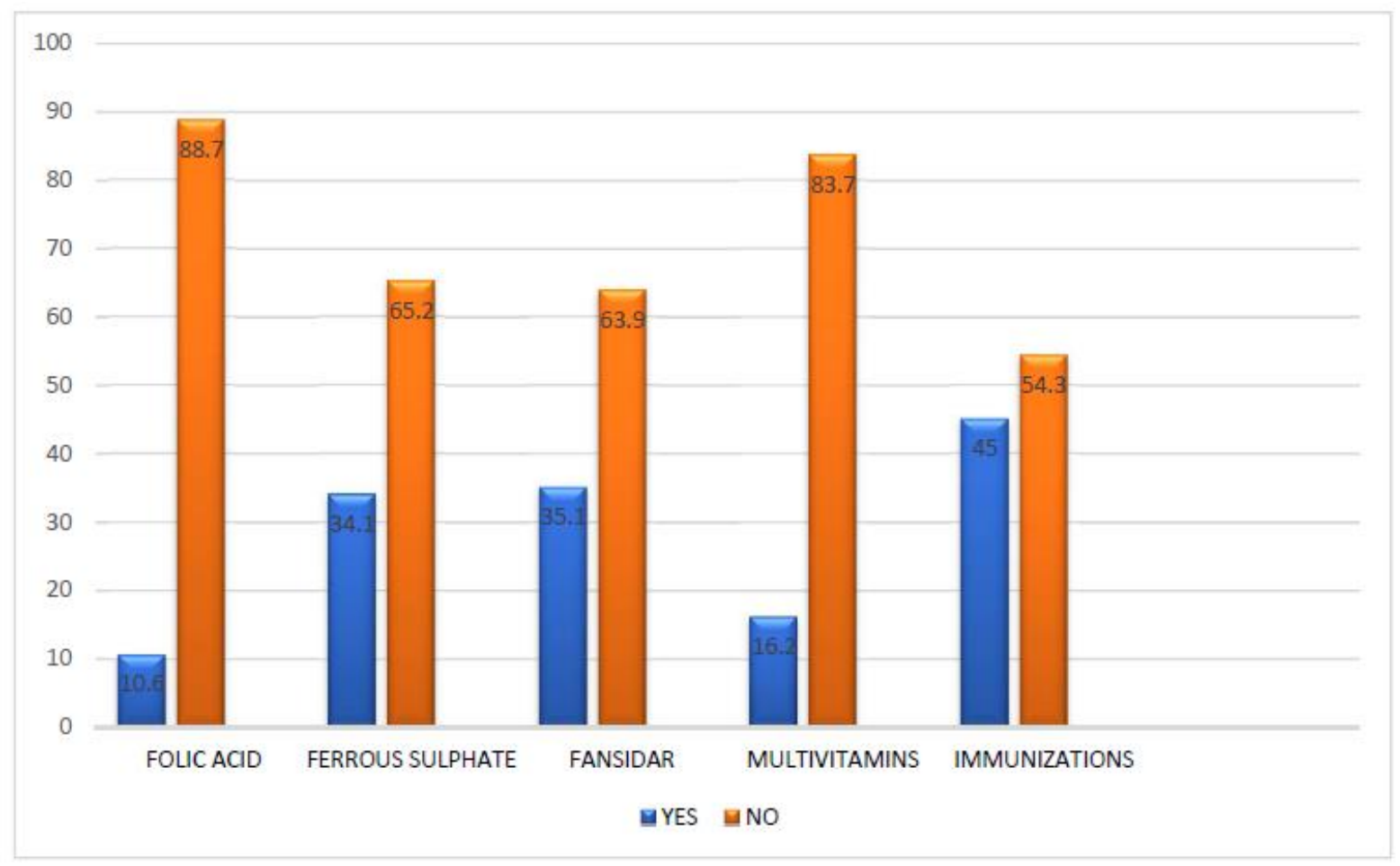

Figure 1. Medications taken to improve health before pregnancy

\section{Vaccinations done before pregnancy}

One hundred and thirty-six (45.0\%) agreed to have been done vaccinations before pregnancy while $\mathrm{n}=164(54.3 \%)$ did not have any immunisations administered to them. Over half $(\mathrm{n}=171 ; 56.6 \%)$ had Tetanus Toxoid Vaccine (TTV) administered to them while $n=131$ (43.4\%) did not have TTV. Only $\mathrm{n}=6$ (2.0) of the respondents had been vaccinated of cancer of the cervix while an overwhelming majority ( $\mathrm{n}=296 ; 98.0 \%)$ were not vaccinated from cancer. Only $\mathrm{n}=5(1.7 \%)$ of the respondents were vaccinated from pneumonia but most of the respondents $n=296$ (98.3\%) were not done (see Table 5.6 and Figure 5.20). 
DOI: $10.21522 /$ TIJPH.2013.06.03.Art014

ISSN: $2520-3134$

120

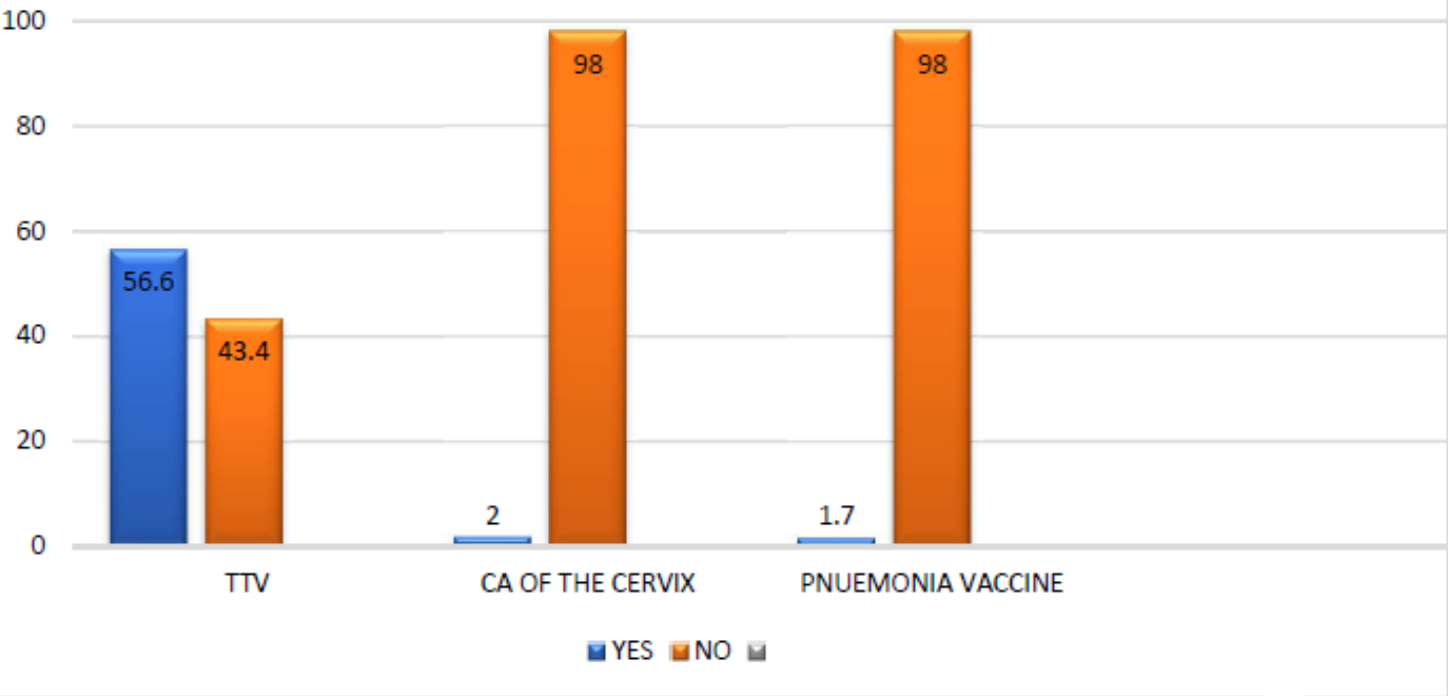

Figure 2. Vaccines given before pregnancy

\section{Health promotion messages on preconception care}

About $n=124$ (41.1\%) got the message that one should be in good health before conception but $n=177$ $(58.6 \%)$ did not know that couples need to be in good health before conception. Over half $n=159$ $(52.6 \%)$ of the respondents were aware that laboratory assessments should done to detect conditions that can be treated before conception while $n=142(47.0 \%)$ were not aware of that message. One hundred thirty-one $(43.4 \%)$ heard that pregnancies need to be planned while $n=170(56.3 \%)$ did not know anything about planning for pregnancy. Message on HTSP was known by $n=75(24.8 \%)$ of the respondents while $\mathrm{n}=226(78.6 \%)$ did not know on healthy timing of pregnancy. One hundred and nineteen $(65.9 \%)$ knew about preparation to have a healthy pregnancy while $n=103(34.3 \%)$ did not know that one need to prepare to have a healthy pregnancy. Refer Table 4 and Figure 5. 
Texila International Journal of Public Health Volume 6, Issue 3, Sep 2018

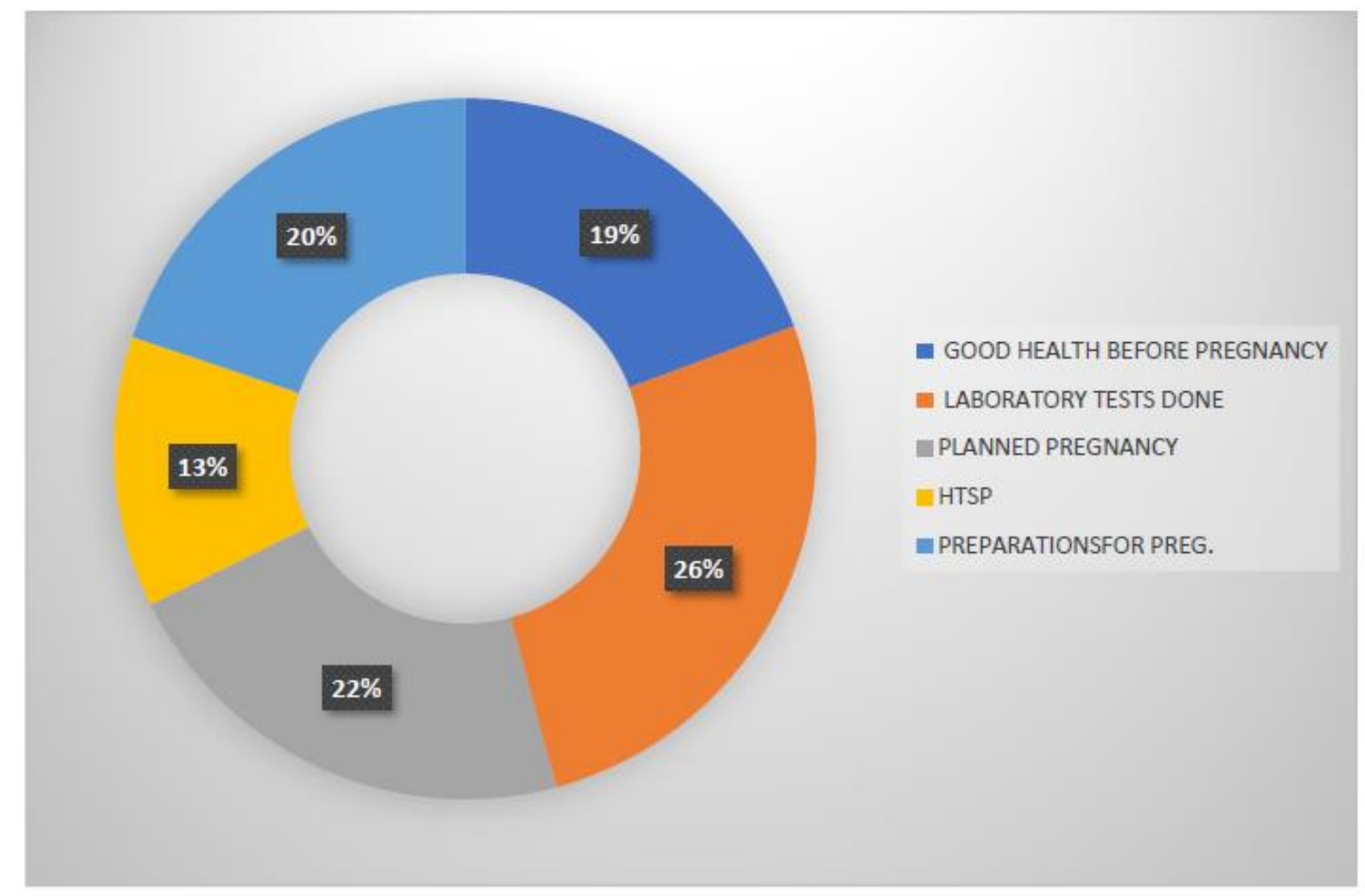

Figure 3. Health promotion messages received

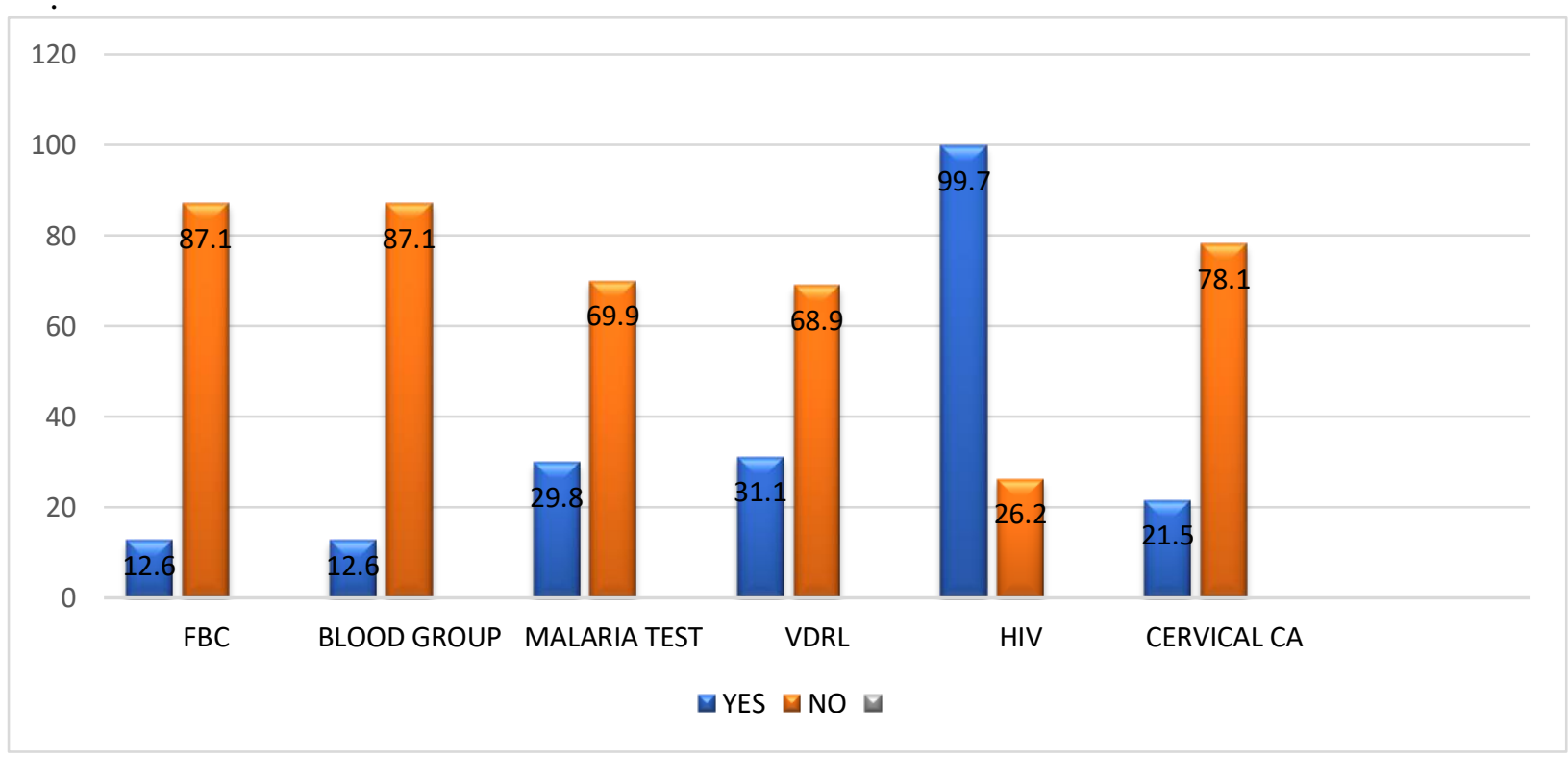

Figure 4. Investigations done before pregnancy 
DOI: $10.21522 / \mathrm{TIJPH} .2013 .06 .03 . A r t 014$

ISSN: 2520-3134

Table 2. Risk assessment done on respondents before conception in relation to their education level

\begin{tabular}{|c|c|c|c|c|c|c|c|c|c|}
\hline \multirow{3}{*}{ Risk assessment } & \multirow{3}{*}{$\begin{array}{c}\text { Yes } \\
\text { n } \\
(\%)\end{array}$} & \multirow{3}{*}{$\begin{array}{c}\text { No } \\
\text { n }(\%)\end{array}$} & \multirow{3}{*}{$\begin{array}{c}\text { Statistic } \\
\text { Kruskal } \\
\text { Wallis test } \\
\left(\mathbf{X}^{2}\right)\end{array}$} & \multirow{3}{*}{$\mathbf{p}$} & \multicolumn{3}{|c|}{ Education } & \multirow{2}{*}{ None } & \multirow{2}{*}{ Total } \\
\hline & & & & & Tertiary & Secondary & Primary & & \\
\hline & & & & & Mean & Mean & Mean & Mean & Mean \\
\hline $\begin{array}{c}\text { Respiratory } \\
\text { problems }\end{array}$ & $\begin{array}{c}96 \\
(32) \\
\end{array}$ & $204(68)$ & 7.33 & .06 & $.34 \pm .48$ & $.24 \pm .43$ & $.39 \pm .49$ & $.33 \pm .58$ & $.32 \pm .47$ \\
\hline Cardiac disease & 33 & 267 & 10.097 & $.02 *$ & $28+.46$ & $.11+.31$ & $.08+.27$ & .00 & $.11+.31$ \\
\hline Hypertension & $\begin{array}{c}96 \\
(32) \\
\end{array}$ & $204(68)$ & 13.823 & $.003 *$ & $.62 \pm .49$ & $28 \pm .45$ & $.28 \pm .45$ & $.33 \pm .58$ & $.32 \pm .47$ \\
\hline Anaemia & $\begin{array}{c}101 \\
(33.6) \\
\end{array}$ & $\begin{array}{c}199 \\
(63.3) \\
\end{array}$ & 15.271 & $.002 *$ & $.62 \pm .49$ & $.26 \pm .44$ & $.34 \pm .48$ & $.67 \pm .58$ & $.34 \pm .47$ \\
\hline Malnutrition & $\begin{array}{c}77 \\
(25.6) \\
\end{array}$ & $\begin{array}{c}223 \\
(74.3) \\
\end{array}$ & 2.376 & .5 & $.31 \pm .47$ & $28 \pm .45$ & $.23 \pm .42$ & $.00 \pm .00$ & $.26 \pm .44$ \\
\hline $\begin{array}{c}\text { Abdominal } \\
\text { surgery }\end{array}$ & $15(5)$ & $\begin{array}{l}285 \\
(95) \\
\end{array}$ & 10.239 & $.02 *$ & $17 \pm .38$ & $.04+.19$ & $.04 \pm .19$ & $.00 \pm .00$ & $.05 \pm .22$ \\
\hline $\begin{array}{c}\text { Gynaecological } \\
\text { problem }\end{array}$ & $\begin{array}{c}52 \\
(17.3) \\
\end{array}$ & $\begin{array}{c}248 \\
(82.6) \\
\end{array}$ & 8.263 & $.04 *$ & $.28 \pm .46$ & $.16+.37$ & $.14 \pm .35$ & $.67 \pm .58$ & $.17 \pm .38$ \\
\hline $\begin{array}{c}\text { Reproductive } \\
\text { health }\end{array}$ & $\begin{array}{c}59 \\
(19.6) \\
\end{array}$ & $\begin{array}{c}241 \\
(80.3) \\
\end{array}$ & 18.734 & $<.001 *$ & $.45 \pm .51$ & $.18 \pm .38$ & $.14 \pm .35$ & $.67 \pm .58$ & $.19 \pm .4$ \\
\hline Diabetes & $\begin{array}{c}30 \\
(10) \\
\end{array}$ & $\begin{array}{l}270 \\
(90) \\
\end{array}$ & 11.391 & $.01 *$ & $.28 \pm .46$ & $.09 \pm .28$ & $.08 \pm .27$ & $.00 \pm 00$ & $.1 \pm .3$ \\
\hline
\end{tabular}

$\mathrm{X}^{2}=$ Chi square, $\mathrm{p}=$ probability, $*=$ significant result, $\mathrm{n}=$ subsample 


\section{Relationship of level of education of respondents to risk assessments done before pregnancy}

Table above displays the results that compare the assessments done in relation to the level of education. Kruskal Wallis test was conducted and showed a significant difference $\left(x^{2}=10.097, p(0.02)\right.$ relationship was found on cardiac disease risk assessment which was conducted in $n=33(10.9 \%)$ of the respondents and indicated a relationship to level of education. Total mean for assessment on cardiac diseases was $.32 \pm .47$, those with primary education's mean was above the total mean and it was $39 \pm 49$, second was those with tertiary education that had a mean of $34 \pm 48$, those with no education's mean was also above the total mean and it was $33 \pm 58$ and lastly those with secondary education's mean was below the total mean and it was $24 \pm 43$. Education has significant relationship with cardiac assessment.

Assessments for risk for cardiac diseases, hypertension, aneamia, diabetic, reproductive health and gynaecological problems showed various statistical significant difference with different levels of education. Those with education indicated to be aware to do various assessments.

\section{Discussion of research results}

Some of the components of PCC are management of chronic diseases, vaccinations, nutrition management and screening and treatment of infections. All these interventions are vital to improve health outcomes for the mother and the baby before conception. The research results indicated gaps on PCC that is provided in Blantyre urban, Malawi. It is important that there should be awareness throughout the health continuum that PCC would improve the health of the mother and the baby if it is done before pregnancy. Health care providers in various health facilities need to initiate health promotion activities like health assessments on all prospective parents to minimize risks that would occur due to diseases and conditions that would be controlled.

\section{Development of preconception guidelines in Malawi}

Guidelines provided by Centre for Disease Control of USA was used to identify the specific information that men and women of child bearing age would need to have PCC before pregnancy. The following interventions were identified through the study and consensus of stakeholders in Malawi.

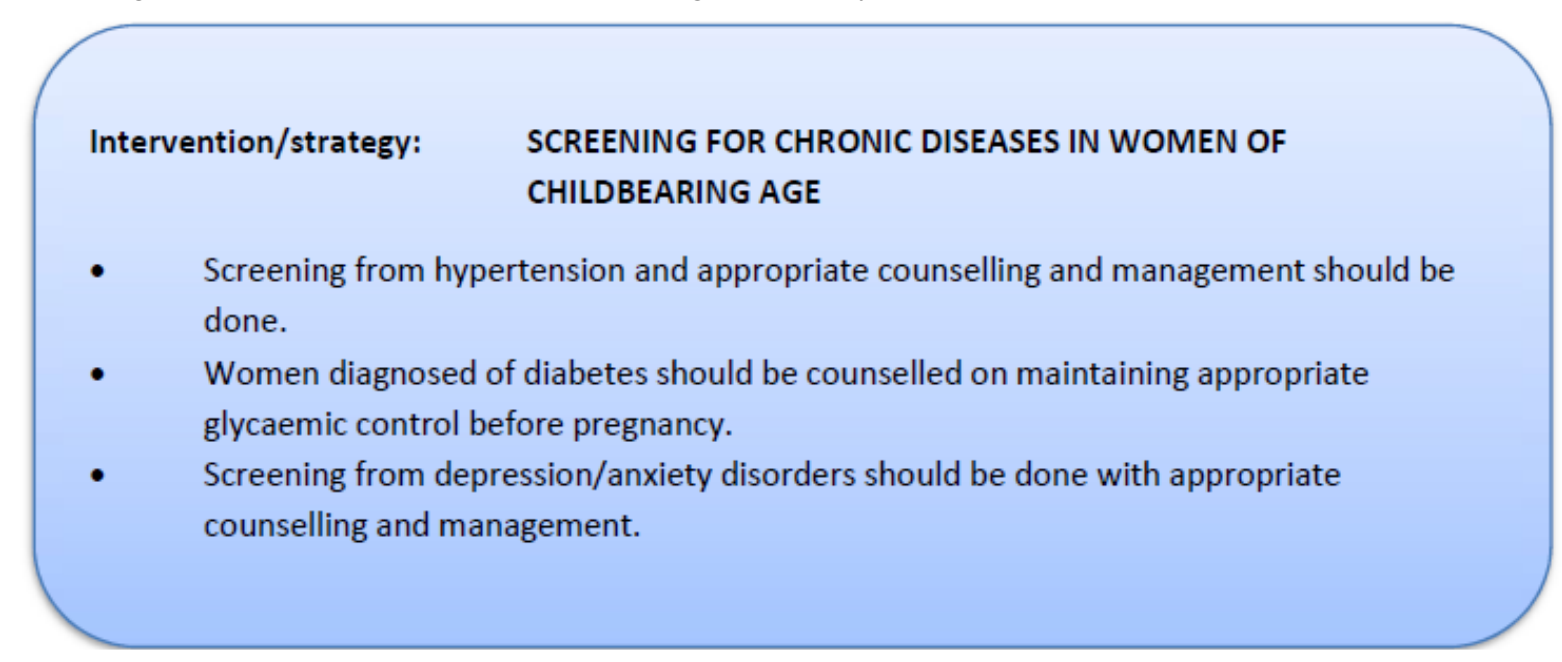

\section{Assessment and maintenance of nutrition}

Good maternal nutrition plays an important role in promoting health of the mother and the baby (Lu 2006). Mothers need to be assessed for low or high body mass index, anaemia, and under-nutrition. Some of the contributing factors of under-nutrition that is common in Malawi are poor dietary patterns, lack of balanced food, eating disorders and lack of supply of multivitamins and folate supplementation. Folic acid $400 \mathrm{mg}$ is a standard preconception recommendation to prevent neural tube defect and iron $12 \mathrm{mg}$ is routinely prescribed and taken before and after pregnancy (Godfrey et al 2017). Anon (2017) said that preconception councelling assist women of the reproductive age to use folic acid before 
DOI: $10.21522 /$ TIJPH.2013.06.03.Art014

ISSN: $2520-3134$

pregnancy to reduce risks of NTDs. In Malawi, folic acid supplementation has not been started despite the recommendations that neural tube defects are controlled.

\section{Intervention/strategy: NUTRITION DURING PRECONCEPTION PERIOD}

- All women with a BMI greater than or equal to $30 \mathrm{~kg} / \mathrm{m}^{2}$ or less than $18.5 \mathrm{~kg} / \mathrm{m}^{2}$ should be counselled about infertility risk and risks during and after pregnancy.

- $\quad$ All women of reproductive age should be advised to take folic acid and to consume a balanced, healthy diet of folate-rich foods. Women at high risk for NTDs should take higher levels of folic acid.

- Laboratory tests results for haemoglobin content, blood group and malaria parasites tests should be conducted before pregnancy.

\section{Assessment and treatment of infections in pre-pregnancy period}

A study conducted by Lassi et al (2014) report that preconception behaviour interventions significantly decline reinfection or new STI $r$ VDRL test is done routinely in Malawi during the antenatal period to identify and treat infections to minimise the transmission of the infections to the baby. In the study, only $31.1 \%$ of the respondents did VDRL test. Those who had messages to have laboratory tests done on haemoglobin content, HB, STIs, blood group and HIV/AIDS test were 52.6\%. Men and women have some knowledge to have laboratory tests but services are not available in the facility for those that are not pregnant.

\section{Intervention/strategy: PREVENTION AND TREATMENT OF INFECTIONS}

- $\quad$ For all women of childbearing age and their partners, assess STI risk, provide counselling and immunisations as indicated to prevent acquisition of STIs, and provide indicated STI testing and treatment.

- $\quad$ Provider Initiated Counceling and Testing (PICT) and Voluntary Testing and councelling (VCT) for HIV/AIDS, initiate ART for PMTCT to have a healthy mother and baby

- Screening and treatment of other infection like periodontal diseases that affect pregnancy

- Immunization status for tetanus, cervical cancer and pneumonia should be reviewed annually and updated as indicated.

\section{Recommendations}

There should be availability of wellness care clinics where individuals, couples and families should access to have the information and care on PCC. Health assessments should be done to men and women of child bearing age to rule out factors that would have adverse effects on pregnancy. Some of the PCC interventions and information should be provided during any encounter with clients that come to the health care facility like antenatal care, under five clinics, family planning clinic and postnatal clinic to create awareness on PCC. Provision of PCC should include adolescents and adults to improve the health of the mother and the baby. The same study should be done in Malawi at a large scale to initiate PCC in the country. 


\section{Limitations of the study}

The study was conducted in only three clinics of the southern part of Malawi in an urban and semiurban location despite that the majority of Malawian population being concentrated more in rural areas. Time and financial constraints limited the researcher to conduct the study at a larger scale as the study was conducted to fulfil the requirements of pursuing a doctor of philosophy degree in health studies for Unisa. However, the findings cannot be generalised to all the Malawian population to have the factors that could influence the provision of PCC. A study at a large scale should be done.

\section{Conclusions}

The study findings indicate that men and women of child bearing age did not have adequate knowledge on doing assessments before becoming pregnant. Most of the respondents did not have the information on assessments of risks before conception. Risks assessments assist women to know the health care information that would assist them to have a healthy mother and baby before pregnancy. Health care measures like having a nutritious diet, controlling the glycemic levels and controlling of weight and blood pressure before pregnancy would assist clients to have a better outcome of a healthy baby and a healthy mother after pregnancy. The guidelines on PCC should include assessments that should be done before one decides to have a pregnancy and interventions should be done to treat the conditions that have adverse effects to pregnancy. There should be awareness to all men and women of child bearing age to have assessments and treat any problem before the onset of pregnancy.

\section{Acknowledgements}

The study would not have been done without the participants that gave consent to participate in the study. The data collection team and the statistician did a recommendable job to have the results of the study. UNISA and COMREC ethical committee offered guidance to refine the study and offered approval for the study to be done. Special gratitude to the District Health Officer for Blantyre urban for allowing the study to be done in the health centres and Queen Elizabeth Central Hospital. A questionnaire from a study done in Texas was adapted to refine on the content that would be examined in research. Special recognition to Prof Modiba for her tireless support to have this study done.

\section{Reference}

[1].Andale. 2012. Slovin's Formula: What is it and when do I use it? [Online]. Available at: http://www.statisticshowto.com/how-to-use-slovins-formula/ (accessed 20 July 2015).

[2].Anderson, J, Ebrahim, S, Floyd, L \& Atrash, H. 2006. Prevalence of risk factors for adverse pregnancy outcomes during pregnancy and the preconception period - United States, 2002-2004. Maternal and Child Health Journal 10(6):575-575.

[3].Anon. 2017. What you need to know counselling patients on PCC: Folate and beyond. [Online]. Available at: https://www.pcc.edu/resources/counseling/:// (accessed 19 March 2017).

[4].Ayalew, Y, Mulat, A, Dile, M \& Simegn, A. 2017. Women's knowledge and associated factors in PCC in adet, west gojjam, northwest Ethiopia: A community based cross sectional study. Reproductive Health 14(1):110.

[5].Bryman, A. 2012. Social research methods. New York. Oxford University Press.

[6].Bombard, J, Robbins, C, Dietz, P \& Valderrama, A. 2013. PCC: The perfect opportunity for health care providers to advise lifestyle changes for hypertensive women. American Journal of Health Promotion 27(sp3): S43-S49.

[7].Curtis, M, Abelman, S, Schulkin, J, Williams, J \& Fasset, E. 2006. Do we practice what we preach? A review of actual clinical practice with regards to PCC guidelines. Maternal and Child Health Journal 10(S1):53-58.

[8].Dean, S, Rudan, I, Althabe, F, Webb Girard, A, Howson, C, Langer, A, Lawn, J, Reeve, M, Teela, K, Toledano, M, Venkatraman, C, Belizan, J, Car, J, Chan, K, Chatterjee, S, Chitekwe, S, Doherty, T, Donnay, F, Ezzati, M, Humayun, K, Jack, B, Lassi, Z, Martorell, R, Poortman, Y \& Bhutta, Z. 2013. Setting research priorities for PCC in low- and middle-income countries: Aiming to reduce maternal and child mortality and morbidity. PLoS Med 10(9): e1001508 (accessed 10 October 2017). 
DOI: 10.21522/TIJPH.2013.06.03.Art014

ISSN: $2520-3134$

[9].Dunlop, A, Logue, K, Thorne, C \& Badal, H. 2013. Change in women's knowledge of general and personal preconception health risks following targeted brief counselling in publicly funded primary care settings. American Journal of Health Promotion 27(sp3): S50-S57.

[10]. Elsinga, J, Van der Pal-de Bruin, K, Le Cessie, S, De Jong-Potjer, L, Verloove-Vanhorick, S \& Assendelft, W. 2006. Preconception counselling initiated by general practitioners in the Netherlands: Reaching couples contemplating pregnancy [ISRCTN53942912]. BMC Family Practice 7(1):1-8.

[11]. Gardiner, P, Hempstead, M, Ring, L, Bickmore, T, Yinusa-Nyahkoon, L, Tran, H, Paasche-Orlow, M, Damus, K \& Jack, B. 2013. Reaching Women through Health Information Technology: The Gabby Preconception Care System. American Journal of Health Promotion 27(sp3): eS11-eS20.

[12]. Godfrey, K, Cutfield, W, Chan, S, Baker, P \& Chong, Y. 2017. Nutritional intervention preconception and during pregnancy to maintain healthy glucose metabolism and offspring health ("NiPPeR"): Study protocol for a randomised controlled trial. Trials 18(1):1-12.

[13]. Lassi, Z, Imam, A, Dean, S \& Bhutta, Z. 2014. Preconception care: screening and management of chronic disease and promoting psychological health. Reproductive Health 11(Suppl 3): S5, 1-20.

[14]. Lu, M, Kotelchuck, M, Culhane, J, Hobel, C, Klerman, L \& Thorp, J. 2006. PCC between Pregnancies: The Content of Internatal Care. Maternal Child Health Journal 10(S1):107-122.

[15]. Kothari, C. 2004. Research methodology. New Delhi: New Age.

[16]. Mason, E, Chandra-Mouli, V, Baltag, V, Christiansen, C, Lassi, Z \& Bhutta, Z. 2014. PCC: Advancing from 'important to do and can be done' to 'is being done and is making a difference'. Reproductive Health 11(Suppl 3): 1-9.

[17]. Mahmud, M \& Mazza, D. 2010. PCC of women with diabetes: a review of current guideline recommendations. BMC Women's Health 10(1):1-7.

[18]. Polit, D, Beck, C \& Polit, D. 2012. Resource manual for nursing research. Philadelphia: Wolters Kluwer Health/Lippincott Williams \& Wilkins.

[19]. Poels, M, Koster, M, Franx, A \& Van Stel, H. 2017. Healthcare providers' views on the delivery of preconception care in a local community setting in the Netherlands. BMC Health Services Research 17(1):1-10.

[20]. Posner, S, Johnson, K, Parker, C, Atrash, H \& Biermann, J. 2006. The national summit on preconception care: A summary of concepts and recommendations. Maternal and Child Health Journal 10(S1):199-207.

[21]. Sagel, U., Krämer, A. \& Mikolajczyk, R. (2011). Incidence of maternal Toxoplasma infections in pregnancy in Upper Austria, 2000-2007. BMC Infectious Diseases, 11(1):1-7.

[22]. Salihu, H, Salinas, A \& Mogos, M. 2012. The Missing Link in Preconceptional Care: The Role of Comparative Effectiveness Research. Maternal and Child Health Journal 17(5):776-782.

[23]. Swaleh, R, Zeng, L, Mbuagbaw, L \& Morrison, K. 2015. Outcomes in the offspring of mothers with prediabetes during pregnancy: a protocol for a systematic review. Systematic Reviews 4(1):1-5.

[24]. Steel, A, Lucke, J \& Adams, J. 2015. The prevalence and nature of the use of preconception services by women with chronic health conditions: an integrative review. BMC Women's Health 15(1):1-12.

[25]. Tuomainen, H, Cross-Bardell, L, Bhoday, M, Qureshi, N and Kai, J. 2013. Opportunities and challenges for enhancing preconception health in primary care: qualitative study with women from ethnically diverse communities. BMJ Open 3(7):1-9.

[26]. Vinturache, A, Moledina, N, McDonald, S, Slater, D \& Tough, S. 2014. Pre-pregnancy Body Mass Index (BMI) and delivery outcomes in a Canadian population. BMC Pregnancy and Childbirth 14(1):1-10.

[27]. Waggoner, M. 2012. Motherhood preconceived the emergence of the preconception health and health care initiative. Journal of Health Politics, Policy and Law 382:345-371.

[28]. World Health Organization. 2015a. WHO/Maternal mortality. [Online]. Available at: http://www.who.int/gho/maternal_health/mortality/maternal_mortality_text/en/ (accessed 14 February 2015). 